\title{
Using 3 Dimension Health Vegetation Index Point Clouds to Determine HLB Infected Citrus Trees
}

\author{
Joseph Cerreta \\ Embry-Riddle Aeronautical University, cerretaj@erau.edu \\ Ashley Hanson \\ Embry-Riddle Aeronautical University, hansona5@my.erau.edu \\ Julianna E. Martorella \\ Embry-Riddle Aeronautical University, martorej@my.erau.edu \\ Stacy Martorella \\ Embry-Riddle Aeronautical University, martores@erau.edu
}

Follow this and additional works at: https://commons.erau.edu/jaaer

Part of the Agricultural Economics Commons, Agricultural Education Commons, and the Science and Technology Studies Commons

\section{Scholarly Commons Citation}

Cerreta, J., Hanson, A., Martorella, J. E., \& Martorella, S. (2018). Using 3 Dimension Health Vegetation Index Point Clouds to Determine HLB Infected Citrus Trees. Journal of Aviation/Aerospace Education \& Research, 28(1). https://doi.org/10.15394/jaaer.2018.1776

This Article is brought to you for free and open access by the Journals at Scholarly Commons. It has been accepted for inclusion in Journal of Aviation/Aerospace Education \& Research by an authorized administrator of Scholarly Commons. For more information, please contact commons@erau.edu. 


\section{Introduction}

The Florida agriculture industry needs innovative methods to detect citrus greening or Huanglongbing (HLB) disease in citrus trees. Researchers have examined health vegetation indices to identify variations in crop health from Unmanned Aircraft Systems (UAS) imagery (Hunt et al., 2010; Zhang \& Kovacs, 2012). Indices can be used in ground-truthing processes to identify areas of infestation and diseased crops. Garcia-Ruiz et al. (2013) investigated using UAS to collect multispectral imagery to detect citrus greening.

A Parrot Sequoia multispectral camera as depicted in Figure 1, flown from UAS, captured narrow-band multispectral images to detect variations within non-suspected HLBinfected (healthy) and HLB-infected citrus trees. Red and near-infrared color bands collected data at $660 \mathrm{~nm}+/-20 \mathrm{~nm}$ and $790 \mathrm{~nm}+/-20 \mathrm{~nm}$ wavelengths respectively. The two bands were used to calculate a Normalized Difference Vegetation Index (NDVI) to determine plant health. Three-dimensional (3D) point clouds from red and near-infrared reflectance images converted into vegetation health indices of citrus groves provided NDVI classifications to identify regions of varying health in citrus crops using the structure of each tree. Three-dimensional NDVI point clouds could enable citrus producers to determine the extent of HLB infections on individual trees differently than two-dimensional (2D) index maps. The use of UAS to collect aerial imagery provides several advantages, including low cost, low altitude flight below cloud layers, and the repeatability of autonomous flight control systems to perform aerial surveying missions (Trujillo, McAdaragh, Ghatas, Burdette, \& Comstock, 2014). 


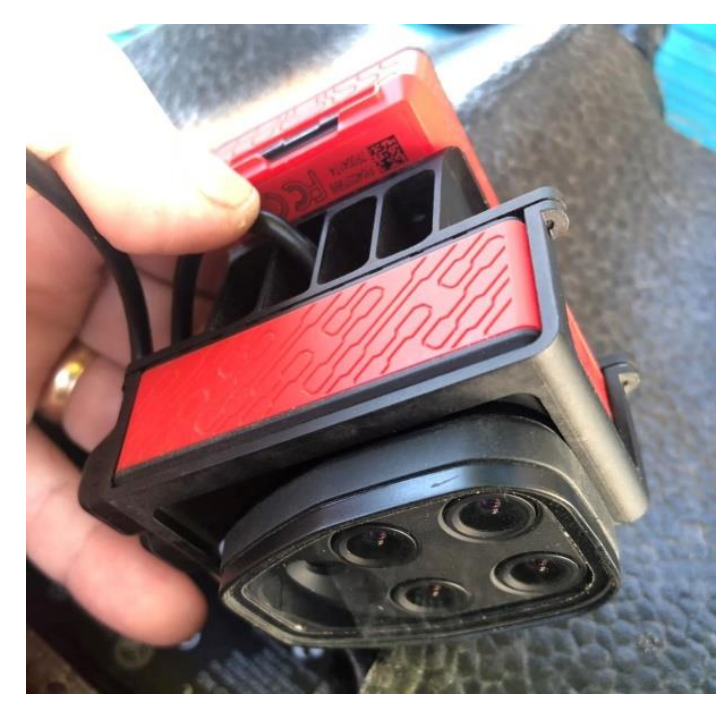

Figure 1. Parrot Sequoia Multispectral Camera.

Three-dimensional point clouds from red and near-infrared reflectance images converted into vegetation health indices of citrus groves provided NDVI classifications to identify regions of varying health in citrus crops using the structure of each tree. Previous work by researchers in the Whitaker Forest sequoia tree project developed by Pix4D (2016) showed vertically-oriented structures, such as trees, can be modeled into a three-dimensional vegetation index point clouds. Three-dimensional vegetation index point clouds could enable citrus producers to determine the extent of HLB infections on individual trees more effectively than two-dimensional index maps and use this information during treatment decisions of the grove.

Similar to Xiao and McPherson (2005), two scales were used to examine differences between trees. A pixel level scale consisted of 3D points in a point cloud or pixels in the 2D reflectance map. A tree level scale consisted of a shapefile surrounding the tree crown, grouping all the points or pixels within the boundary of the crown. A Whole-Tree Vegetation Index (WTVI) (S. Klinke, personal communication, June 26, 2018) was caculated as a ratio of pixels within five NDVI classifications ranging from $0.0-0.2,0.2-0.4,0.4-0.6,0.6-0.8$, and $0.8-1.0$. 
Three-dimensional WTVI data was compared to 2D WTVI data to determine if differences existed.

\section{Research Objectives}

The objective of this research was to determine if any differences exist between 3D NDVI point clouds and traditional 2D NDVI reflectance maps for detecting healthiness variations of suspected HLB-infected citrus trees with images collected from a UAS. Photogrammetric image processing algorithms were used to develop 3D NDVI point clouds, and 2D NDVI reflectance maps. This research project examined two research questions: 1) To what extent, if any, can a 3D NDVI point cloud determine healthiness variation of suspected HLBinfected citrus trees?, and 2) How do 3D NDVI point clouds compare to 2D NDVI reflectance maps for detecting differences in healthiness of suspected HLB-infected citrus trees?

\section{Study Area}

\section{Materials and Methods}

The study area was a 255-acre orange grove, located in DeLeon Springs, Florida, United States. This area consisted of a humid subtropical climate with an average of 53 inches of rainfall within 72 precipitation days per year. The field elevation was 48 feet mean sea level.

\section{Sample Population}

For this study, the researchers examined a 30-acre section containing a total of 3,258

trees. The sample size was determined using a confidence level of $95 \%$ with a confidence interval of $11 \%$. The calculated sample size was 79 trees. Based on work purported by Sankaran, Maja, Buchanon, and Ehsani (2013), trees with symptoms of HLB disease included chlorosis and blotchy mottle and were visually inspected via ground observations and marked with global positioning system (GPS) coordinates. An additional 79 trees, which did not exhibit symptoms of HLB disease, were marked with GPS as control trees. 


\section{Statistical Analysis and Tools}

Wilcoxon Signed-Rank testing was performed using Minitab (Minitab Inc., Version 18, State College, PA) to compare the differences between 3D WTVI and 2D WTVI paired data for all 79 suspected HLB-infected trees. The Wilcoxon Signed-Rank test was selected because the data was not normally distributed. Researchers conducted a two-sample T-test on the quantity of point cloud points between suspected HLB-infected trees and non-suspected HLB-infected trees. A separate two-sample T-test was performed on the tree crown area between suspected HLBinfected trees and non-suspected HLB-infected trees. These tests were selected to determine differences between means.

\section{Limitations}

This research investigated differences between 3D NDVI point clouds and 2D NDVI reflectance maps at a single citrus grove in central Florida. Other citrus groves may have other variables that may affect the probability of detecting differences. The multispectral data collected for this research were at a specific altitude, airspeed, and overlap percentage. The effects of altitude, airspeed, or overlap percentage on the ability to detect differences were not examined in this research. Since the multispectral data was collected on a single day, atmospheric variation was not experienced. Atmospheric variation, or other environmental factors could have an effect on the probability of detecting differences between 3D NDVI point clouds and 2D NDVI reflectance maps.

\section{Remotely Sensed Data Collection}

Field experiments began on February 19, 2018, with an RGB orthomosaic ground truth map followed by ground observations to mark suspected HLB-infected tree locations. A DJI Mavic Pro UAS captured the ground truth map imagery (DJI Mavic Pro, CP.PT.000500, DJI, 
Shenzhen, China) using DJI Ground Station Pro (DJI Ground Station Pro, Version 1.8.3, DJI Corporation, Shenzhen, China). The Mavic Pro UAS flew over the sample area at an altitude of 301 feet above ground level, resulting in a ground sample distance (GSD) of 1.1 inches per pixel. A 1.1 inch GSD provided a practical balance between UAS battery endurance and resolution necessary for the point cloud generation in Pix4D. The flight, oriented in a North-South grid pattern, had an $80 \%$ longitudinal and $70 \%$ lateral overlap ratio. The Mavic Pro UAS flight captured 241 images and was processed in Pix4Dmapper Pro (Pix4Dmapper Pro, Version 4.1.21, Pix4D, Lausanne, Switzerland).

Citrus trees within the sample area, as depicted in Figure 2, were known to have the HLB disease (T. Tedder, personal communication, February 13, 2018). Researchers used visual ground observations throughout the sample area to mark suspected HLB-infected trees present. Upon observing a suspected HLB-infected tree, a GPS location marked the coordinates of the tree for image processing. Non-suspected HLB-infected trees were also marked using the RGB orthomosaic map and GPS coordinates. The GPS accuracy was determined to be 9 feet from within the GPS itself; however, since the suspected HLB-infected tree crown averaged $188 \mathrm{ft}^{2}$, error in GPS positioning while marking trees was not deemed a risk of marking the wrong tree. 


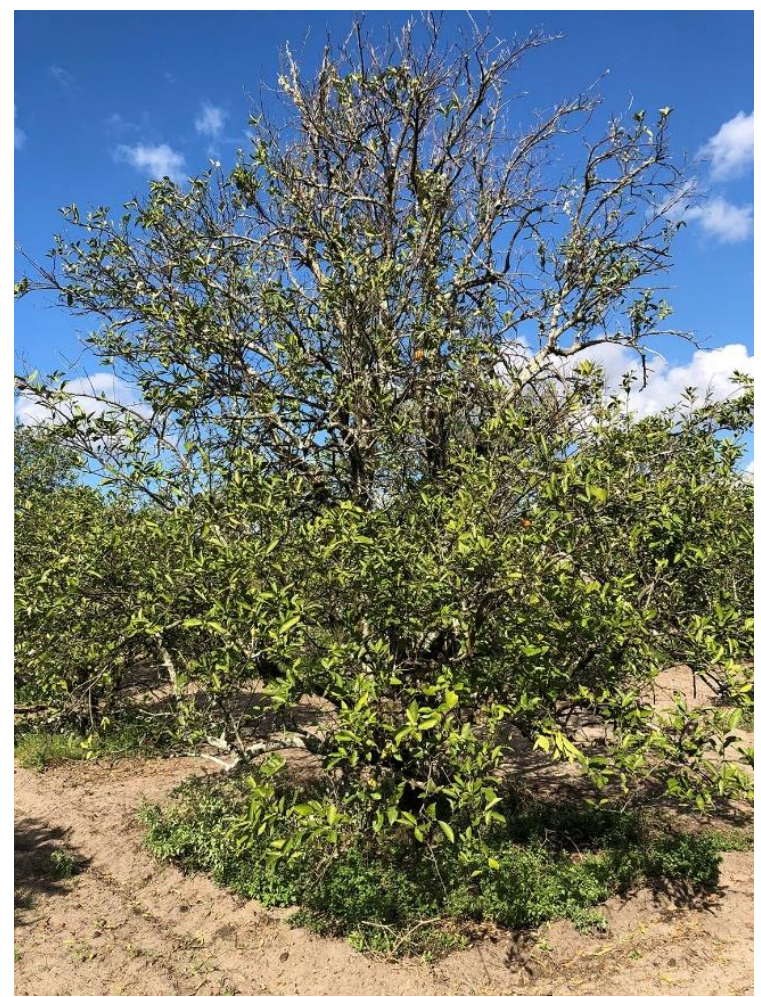

Figure 2. Suspected HLB-infected tree \#6.

The multispectral aerial data collection was performed on February 21, 2018, using a Parrot Sequoia camera flown from a Parrot Disco Pro Ag UAS. Two sequential flights collected the aerial imagery at 11:20 a.m. and 11:39 a.m. Before the aerial data collection, the Parrot Sequoia collected images from an AIRINOV calibrated reflectance target (AIRINOV reflectance target, 502-38-01, AIRINOV Corporation, Paris, France). The known-reflectance images were used in post-processing to radiometrically calibrate images between the two UAS flights as well as the color temperature of natural light at the time of collection (Haghighattalab et al., 2016). Researchers used Pix4Dcapture (Pix4D) to plan two Parrot Disco Pro Ag flights. The sample area was approximately 1,500 feet by 1,500 feet. The flight altitude was planned at 250 feet above ground level, resulting in a GSD of 2.9 inches per pixel. Flight planning parameters were set with a $75 \%$ longitudinal and $65 \%$ lateral overlap ratio, triggering the Parrot Sequoia camera automatically. The Pix4Dcapture software automatically calculated the distance between 
image acquisitions and flight line spacing in a grid pattern. The first of the two multispectral data collection flights was oriented in a grid pattern with an East-West pattern. The second flight was oriented in a grid pattern with a North-South pattern. Together, these two flights resulted in a double grid pattern to collect aerial imagery from all perspectives of each tree in the sample area. Each flight required 16 minutes to complete. The Parrot Sequoia captured 3,024 multispectral images from the two flights.

\section{Data Processing}

Data processing consisted of ground truthing, UAS flight, post-processing of the multispectral images, analysis, and results determination. After completing the two flights, researchers imported all multispectral and calibration target images into Pix4Dmapper Pro (Pix4D). The Ag Multispectral processing options template was used. The Initial Processing was set in the Pix4D processing options at full image scale. The Matching Image Pairs was set to Aerial Grid or Corridor. The targeted number of keypoints was set at 10,000. The alternative calibration method was used with a custom rematch. Camera Internals and Externals, Aerial Triangulation, and Bundle Block Adjustment information were exported.

The red and near-infrared 3D point clouds were imported into Global Mapper GIS software (Global Mapper, Version 19.1, Blue Marble Geographics, Hallowell, Maine). Both red and near-infrared 3D point clouds were transformed into an elevation grid, creating a red and near-infrared elevation grid point cloud. The Color RGB grid type was chosen with automatic grid spacing. Tight elevation grid distance criteria were used. There were no tiling or grid boundaries emplaced.

An NDVI calculation was computed from the transformed red and near-infrared elevation grid, creating a 3D NDVI point cloud. The NDVI predefined formula of (B4 - B1) / (B4 + B1), 
with the B4 and B1 as the NIR and red color channels respectively were used. The NDVI values from the calculation were represented in elevation from -1.0 to +1.0 . The 3D NDVI point clouds enabled calculations at the pixel level scale. See Figure 3 for a depiction of 3D NVDI elevation data.

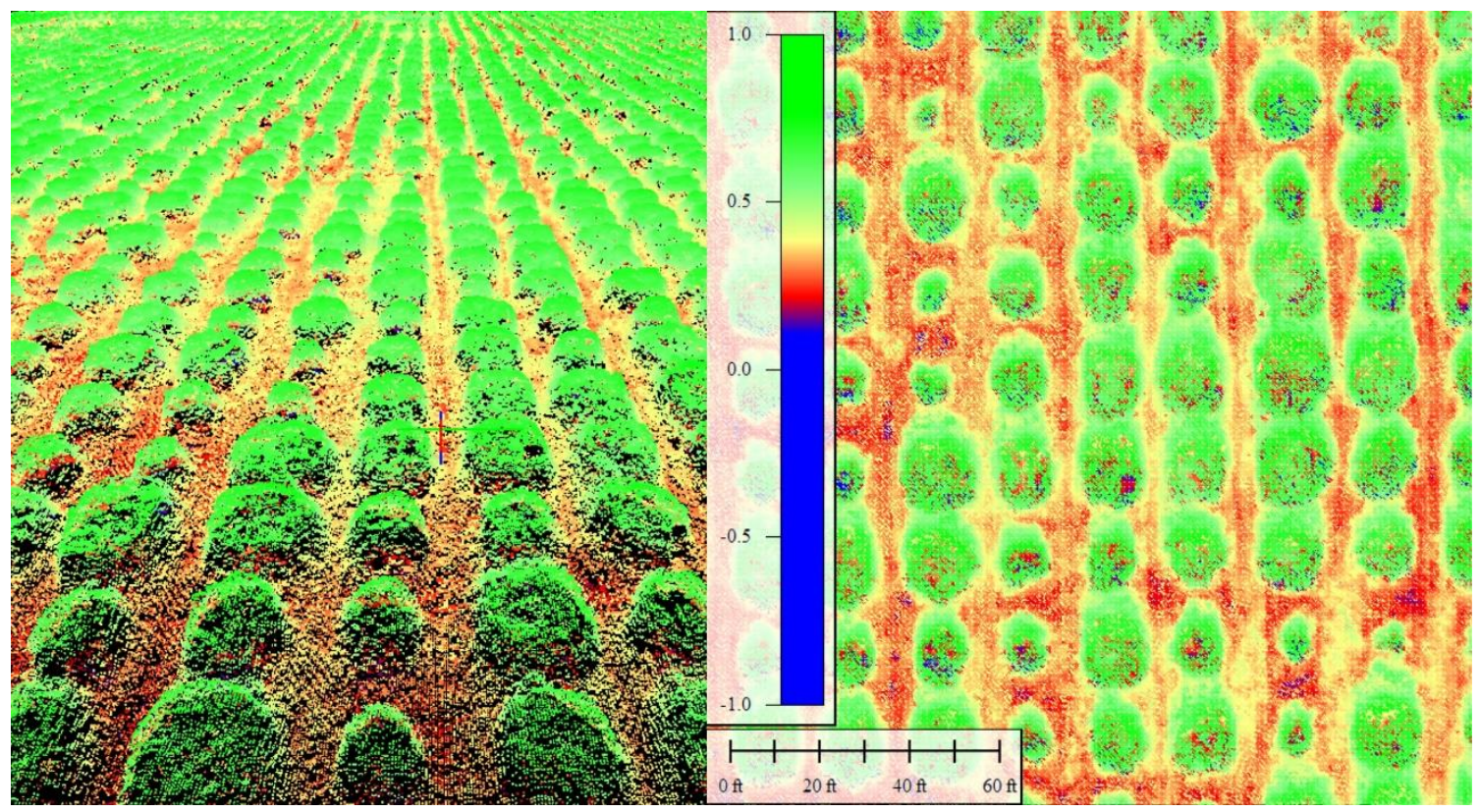

Figure 3. 3D and 2D NDVI elevation dataset in Global Mapper.

The RGB orthomosaic was coregistered to the 3D NDVI point cloud in Global Mapper using 27 control points across the sample area to align coordinates between the dataset layers. The coregistration of layers corrected for variation of GPS antenna-to-camera orientation on the two different UAS and GPS drifts from two different dates images were acquired (Leprince, Barbot, Ayoub, \& Avouac, 2007). The Mavic's RGB orthomosaic used during the ground truthing process provided an additional layer to determine the exact extent of the trees for the creation of shapefiles for each tree crown area.

Within Global Mapper, a shapefile was created around the circumference of each of the 
79 suspected HLB-infected trees and sequentially numbered. A control-tree shapefile was also created around the circumference of each non-suspected HLB-infected tree and sequentially numbered. The shapefiles around each tree enabled WTVI calculations at the tree level scale. Within Pix4D Mapper Pro, each shapefile was examined separately within the Index Calculator. Each shapefile was imported as a region. An NDVI calculation was performed around each shapefile individually. The use of shapefiles aligned the geolocation of shapefiles within both the 3D NDVI dataset in Global Mapper and the 2D NDVI dataset in Pix4Dmapper Pro to examine the same tree and tree crown area of each tree.

The NVDI calculations were classified into five equally spaced proportions from an NDVI value of 0.0 to 1.0. Because the shapefiles were representative of the extent of each tree crown area, negative NDVI values were not observed. The five classifications were designated as shown in Table 1.

Table 1

NDVI Classification

\begin{tabular}{lc}
\hline Classification & False-Color Represented \\
\hline $0.0-0.2$ & Red \\
$0.2-0.4$ & Dark Orange \\
$0.4-0.6$ & Light Orange \\
$0.6-0.8$ & Yellow \\
$0.8-1.0$ & Green
\end{tabular}

Note. NDVI classifications of equal distribution false colorized within the Pix4D index calculator.

The 3D NDVI point cloud was cropped around each shapefile independently. The Global Mapper digitizer tool was used to select each shapefile. The selected area was cropped, to only 
have the 3D NDVI point cloud data from within the shapefile area available. The crop tool marked cropped features as deleted data. The Chart and Area Manager tool was used to calculate the 3D NDVI values within five classifications for only the extent of each shapefile. A histogram was generated to show the distribution of NDVI attribute values transformed to elevation data within the five classifications. The quantity of point cloud points within the five 3D NDVI classifications was recorded on an Excel worksheet. For 3D NDVI data, the quantity of point cloud points was converted into a proportion of the overall quantity of points for each shapefile to align with the distribution method used by Pix4D. See Figure 4 for a depiction of a 3D NDVI point cloud distributed into the five classifications.

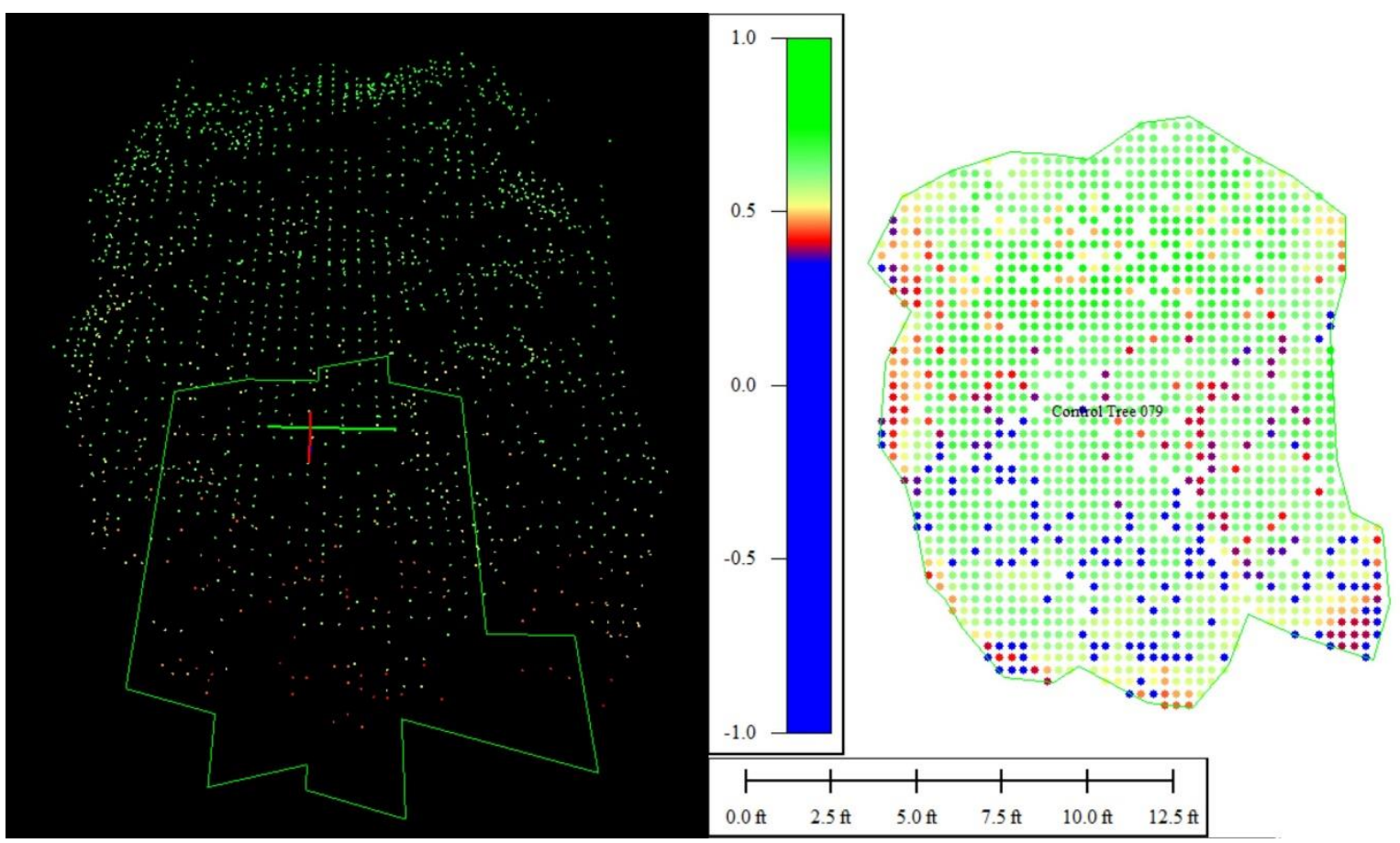

Figure 4. 3D NDVI of non-suspected HLB-infected tree \#79 in Global Mapper. 
The 2D NDVI values were examined from each shapefile representing each tree as a region. The same NDVI mathematical calculation was performed as in Global Mapper to generate a NDVI false-color image with five equally spaced classes. The classifications were set in Pix4D with a minimum range of 0.0 and a maximum range of 1.0. Data was clamped to fit within this range. The distribution of NDVI data of the five 2D NDVI classifications was recorded on an Excel worksheet. See Figure 5 for a depiction of 2D NDVI data distributed in Pix4D's index calculator.

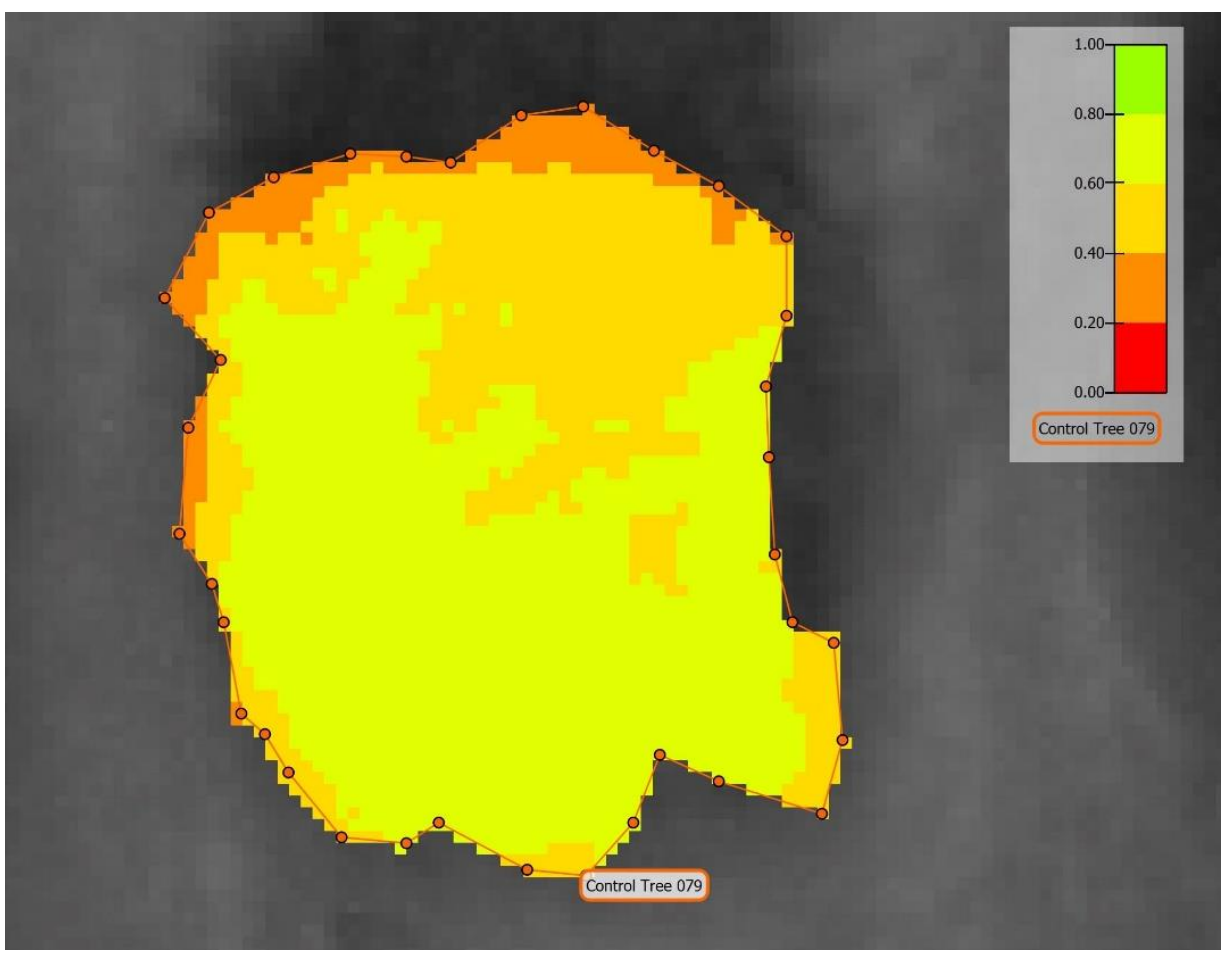

Figure 5. 2D NDVI of a non-suspected HLB-infected tree \#79 in Pix4D.

Each of the five classes of 3D NVDI point cloud and 2D NDVI reflectance proportions were separated into individual columns in Excel. For 3D NDVI data, the quantity of point cloud points was transformed into proportions of the overall quantity of points for each shapefile. Five columns represented the proportion of 3D NDVI classifications ranging from 1) 0.0 to $0.20,2$ ) 
0.2 to $0.4,3) 0.4$ to $0.6,4) 0.6$ to 0.8 , and 5) 0.8 to 1.0 . Five columns also represented the proportions of 2D NDVI classifications.

A shapefile within the boundary of each tree crown grouped the 3D NDVI points from the pointcloud or the NDVI pixels from the $2 \mathrm{D}$ reflectance map into a tree-level scale. A WTVI (S. Klinke, personal communication, June 26, 2018) was calculated as the sum of the proportions for each of the five NVDI classifications with a weighting factor added to each classification. The weighting factor represented the center NDVI value for each classification. For example, the 0.2-0.4 classification had a NVDI center of 0.3 ; 0.3 was used as the weighting factor for the proportion of points or pixels within that classification. Equation 1 depicts the WTVI. The WTVI calculation was applied to each tree, generating a healthiness index value at the tree level. The WHVI was applied to both non-suspected and suspected HLB-infected trees for Wilcoxon Signed-Rank testing to compare the median differences between WHVI values.

$$
\mathrm{WTVI}=\left(p_{1} \times 0.1\right)+\left(p_{2} \times 0.3\right)+\left(p_{3} \times 0.5\right)+\left(p_{4} \times 0.7\right)+\left(p_{5} \times 0.9\right)
$$

Equation 1. WTVI calculation for whole-tree healthiness.

Note: $p_{1}=$ proportion of NDVI points/pixels in the $0.0-0.2$ classification. $p_{2}=0.2-0.4$ classification. $p_{3}=0.4-0.6$ classification. $p_{4}=0.6-0.8$ classification. $p_{5}=0.8-1.0$ classification.

\section{Results}

Five classifications from 79 samples of non-suspected and 79 suspected HLB-infected trees were observed between 3D NDVI and 2D NDVI values. Both 3D NDVI and two dimensional NDVI data came from the same multispectral images and the same red and nearinfrared point cloud datasets. Table 2 depicts the distribution of all suspected and non-suspected HLB-infected trees within the five classifications. 
Table 2

Distribution of Three and 2D NVDI Classifications for All Sampled Trees

\begin{tabular}{lcrrrrr}
\hline Tree Group & $N$ & $0.0-0.20$ & $0.2-0.4$ & $0.4-0.6$ & $0.6-0.8$ & $0.8-1.0$ \\
\hline 3D Suspected HLB-Infected Trees & 79 & 0.24 & 11.97 & 46.10 & 41.69 & 0.00 \\
2D Suspected HLB-Infected Trees & 79 & 0.00 & 7.46 & 84.15 & 8.40 & 0.00 \\
3D Non-Suspected HLB-Infected Trees & 79 & 2.62 & 12.50 & 15.78 & 69.10 & 0.00 \\
2D Non-Suspected HLB-Infected Trees & 79 & 0.00 & 0.00 & 39.96 & 60.04 & 0.00 \\
\hline
\end{tabular}

Note. Values are in $\%$ of the total for each row.

Wilcoxon Signed-Rank testing was performed using Minitab (Minitab Inc., Version 18, State College, PA) to compare the differences between 3D WTVI and 2D WTVI paired data for all 79 suspected HLB-infected trees. An additional 79 non-suspected HLB-infected trees were examined using the same processing workflow and the same five-category classification weighting into a WTVI as the suspected HLB-infected tree sample. Wilcoxon Signed-Rank testing was also performed in Minitab to determine a statistical difference between 3D WTVI and 2D WTVI pairs for all 79 non-suspected HLB-infected trees. Table 3 also depicts the results of Wilcoxon Signed-Rank testing for the suspected and non-suspected HLB-infected trees sampled.

Table 3

Wilcoxon Signed-Rank Test for Differences Between Three- and Two-Dimensional Suspected and Non-Suspected HLB-Infected Trees

\begin{tabular}{lllcl}
\hline Tree Group & $N$ & Dedian of & & \\
\hline 3D - 2D Suspected HLB-Infected Trees & 79 & $-0.0267526^{*}$ & $(-0.0439722,-0.0083131)$ & 0.007 \\
3D - 2D Non-Suspected HLB-Infected Trees & 79 & $-0.105974^{*}$ & $(-0.1190840,-0.0953404)$ & 0.000 \\
\hline
\end{tabular}

Note. Differences are at the tree-level in the WTVI. * indicates $(p<0.05)$. 
The spatial resolution of 3D NDVI points was the same between the suspected HLBinfected trees and the non-suspected HLB-infected trees. The suspected HLB-infected trees had a mean point count of 1,458 points with a mean area of 188.1 square feet. The non-suspected HLB-infected trees had a mean point count of 1,605 points with a mean area of 205.1 square feet. Table 4 depicts the mean 3D NVDI points differences between suspected HLB-infected and non-suspected HLB-infected trees. Table 5 depicts mean tree crown area differences between suspected HLB-infected trees and non-suspected HLB-infected trees.

Table 4

Two-Sample T-Test of 3D NVDI Point Quantity Between Suspected HLB-Infected and NonSuspected HLB-Infected Trees

\begin{tabular}{lllll}
\hline Tree Group & $N$ & Mean & $\begin{array}{l}\text { Std } \\
\text { Dev }\end{array}$ & $\begin{array}{l}\text { Std Error } \\
\text { Mean }\end{array}$ \\
\hline Suspected HLB-Infected Trees & 79 & $1458^{*}$ & 392 & 44 \\
& & & & 403 \\
Non-Suspected HLB-Infected Trees & 79 & $1605^{*}$ & 403
\end{tabular}

Note. Values are in quantity of 3D NVDI points. $P=0.021 . *$ indicates $(p<0.05)$.

Table 5

Two-Sample T-Test of Tree Crown Area Between Suspected HLB-Infected and Non-Suspected HLB-Infected Trees

\begin{tabular}{lllll}
\hline Tree Group & $N$ & Mean & $\begin{array}{l}\text { Std } \\
\text { Dev }\end{array}$ & $\begin{array}{l}\text { Std Error } \\
\text { Mean }\end{array}$ \\
\hline Suspected HLB-Infected Trees & 79 & $188.1^{*}$ & 49.9 & 5.6 \\
Non-Suspected HLB-Infected Trees & 79 & $205.1^{*}$ & 48.8 & 5.5 \\
& & & & \\
\hline Note. $N=158$. Values are in square feet. $p=0.032$. & $*$ indicates $(p<0.05)$.
\end{tabular}




\section{Discussion}

To answer research question one, to what extent, if any, can a 3D NDVI point cloud determine healthiness variations of suspected HLB-infected citrus trees, a WTVI was calculated to represent a tree-level scale health vegetation index from pixel-level scale NDVI data. A WTVI (S. Klinke, personal communication, June 26, 2018) was calculated as the sum of the proportions for each of the five NVDI classifications with a weighting factor added for each classification. Wilcoxon Signed-Rank testing compared the median differences of the WTVI for suspected HLB-infected trees using three- and two-dimensional data. The results indicated significant differences between 3D and 2D points, grouped in the tree level, for suspected HLBinfected trees $(p=0.007)$. The data suggests 3D NDVI point cloud points were more sensitive to less healthy levels of NDVI values by $2.7 \%$ compared to two dimensional NDVI data for suspected HLB-infected trees. Researchers concluded 3D NDVI point clouds could be used to determine healthiness variations in suspected HLB-infected citrus trees.

Examining differences in the non-suspected HLB-infected trees provided similar results for the suspected HLB-infected trees. Wilcoxon Signed-Rank testing compared the median differences of the WTVI for non-suspected HLB-infected trees using three- and two-dimensional data. The results indicated statistical differences between median pairs of 3D NDVI point clouds and 2D NDVI reflectance maps groups in the tree level $(p=0.000)$. The data suggests 3D NDVI point cloud points were more sensitive to less healthy levels of NDVI values by $10.6 \%$ compared to two-dimensional NDVI data for non-suspected HLB-infected trees.

To answer research question two, how do 3D NDVI point clouds compare to 2D NDVI reflectance maps for HLB infected citrus trees, when examining the suspected HLB-infected trees, the 3D NDVI point clouds, shown in Table 3, had a wider distribution across all NDVI 
classifications compared to the 2D NDVI reflectance maps. Additionally, the five-NDVI classification distribution of non-suspected HLB-infected trees also showed a wider distribution with 3D NDVI point clouds compared to 2D NDVI reflectance maps. The vertical structure of the citrus tree may contribute to the different distribution.

Researchers conducted two-sample T-tests on the quantity of point cloud points and tree crown area between suspected HLB-infected trees and non-suspected HLB-infected trees. An increased quantity of 3D NDVI points in a point cloud resulted from an increased amount of reflectance data observed by Pix4D during the point cloud generation process step. There was a $10.01 \%(p=0.021)$ increase in three-dimension NDVI point cloud points for non-suspected HLB-infected trees compared to the suspected HLB-infected trees. Additionally, there was a 9.04\% ( $p=0.032)$ increase in tree crown dimension (square feet) for non-suspected HLBinfected trees compared to suspected HLB-infected trees. These data suggest suspected HLBinfected trees were smaller than non-suspected HLB-infected trees. Three-dimensional NDVI point clouds can determine differences in quantity of points of 3D NDVI point clouds and tree crown dimensions between suspected HLB-infected and non-suspected HLB-infected trees.

\section{Future Research}

Additional research is needed to investigate if differences between 3D NDVI point clouds and 2D reflectance maps at other citrus groves, with varying levels of suspected HLB infections, could be detected. The effects of altitude, airspeed, or overlap percentage on the ability to detect differences were not examined in this research. Atmospheric variation was not experienced in this research because these flights were sequentially performed on the same day with similar conditions between the two flights. Atmospheric variation, or other factors, could have an effect on the probability of detecting differences. 


\section{References}

Garcia-Ruiz, F., Sankaran, S., Maja, J. M., Lee, W. S., Rasmussen, J., \& Ehsani, R. (2013). Comparison of two aerial imaging platforms for identification of huanglongbing-infected citrus trees. Computers and Electronics in Agriculture, 91, 106-115. https://doi.org/10.1016/j.compag.2012.12.002

Haghighattalab, A., González Pérez, L., Mondal, S., Singh, D., Schinstock, D., Rutkoski, J., .. . \& Poland, J. (2016). Application of unmanned aerial systems for high throughput phenotyping of large wheat breeding nurseries. Plant Methods, 12(1), 35. https://doi.org/10.1186/s13007-016-0134-6

Hunt, E. R., Jr., Hively, W. D., Fujikawa, S. J., Linden, D. S., Daughtry, C. S. T., \& McCarty, G. W. (2010). Acquisition of NIR-green-blue digital photographs from unmanned aircraft for crop monitoring. Remote Sensing, 2(1), 290-305. https://doi.org/10.3390/rs2010290

Leprince, S., Barbot, S., Ayoub, F., \& Avouac, J.-P. (2007). Automatic and precise orthorectification, coregistration, and subpixel correlation of satellite images, application to ground deformation measurements. IEEE Transactions on Geoscience and Remote Sensing, 45(6), 1529-1558. https://doi.org/10.1109/TGRS.2006.888937

Pix4D. (2016). An exploratory project: Sequoia trees in 3D NDVI. Retrieved from https://pix4d.com/3d-ndvi/

Sankaran, S., Maja, J. M., Buchanon, S., \& Ehsani, R. (2013). Huanglongbing (citrus greening) detection using visible, near infrared and thermal imaging techniques. Sensors, 13(2), 2117-2130. https://doi.org/10.3390/s130202117 
Trujillo, A. C., McAdaragh, R. M., Ghatas, R. W., Burdette, D. W., \& Comstock, J. R., Jr. (2014). Making the case for new research to support the integration of small unmanned aircraft systems into the national airspace system. NASA/TP-2015-218684, February 2015.

Xiao, Q., \& McPherson, E. G. (2005). Tree health mapping with multispectral remote sensing data at UC Davis, California. Urban Ecosystems, 8(3-4), 349-361. https://doi.org/10.1007/s11252-005-4867-7

Zhang, C., \& Kovacs, J. M. (2012). The application of small unmanned aerial systems for precision agriculture: A review. Precision Agriculture, 13(6), 693-712. https://doi.org/10.1007/s11119-012-9274-5 